\title{
Excited state analysis in the quasi-PDF matrix elements
}

\section{Ruizi Li}

Department of Physics and Astronomy, Michigan State University, East Lansing, MI 48824

E-mail: liruiziepa.msu.edu

\section{Yi-Bo Yang ${ }^{* \dagger}$}

Department of Physics and Astronomy, Michigan State University, East Lansing, MI 48824

E-mail: yangyiboepa.msu.edu

\section{Huey-Wen Lin}

Department of Physics and Astronomy, department of Computational Mathematics, Science and Engineering, Michigan State University, East Lansing, MI 48824

\begin{abstract}
We study and demonstrate the ground-state extrapolation of the unpolarized and polarized nucleon quark quasi-PDF matrix elements in a highly boosted hadron frame on the lattice. The calculation is done using the Wilson clover quark on a MILC's dynamical $N_{f}=2+1+1$ highly improved staggered quarks (HISQ) ensemble with one step hypercubic smearing, and with the lattice spacing $a \approx 0.09 \mathrm{fm}$ and pion mass $310 \mathrm{MeV}$. Applying the Gaussian momentum-smeared quark sources and comparing various fits in 1-, 2-, and 3-state fitting models, we show that excitedstate contributions can be under control in the lattice calculation of the nucleon quark quasi-PDF matrix elements.
\end{abstract}

The 36th Annual International Symposium on Lattice Field Theory - LATTICE2018 22-28 July, 2018

Michigan State University, East Lansing, Michigan, USA.

\footnotetext{
* Speaker.

${ }^{\dagger}$ Current address: Institute of Theoretical Physics, Chinese Academy of Science, Beijing, China
} 


\section{Introduction}

The direct calculation of the parton distribution function (PDF) [1] in the hadron in lattice quantum chromodynamics (LQCD) has been greatly promoted by the recent development of the large momentum effective theory (LaMET) [2]. According to LaMET, the PDF at the full Bjoken$x$ region can be recovered in LQCD from first principles. The recipe includes calculations of the so-called hadron quasi-PDF in a large-momentum hadron frame. This procedure has been shown surpassing traditional PDF calculations in LQCD through a few lowest moments [3], not only with the advanced theory backup but also with improved results that now are compared with experimental data side-by-side. Calculations of the quasi-PDF matrix element on the lattice, however, are nontrivial, and they suffer from poor signal-to-noise ratios with large momentum quark propagators required by LaMET. In this proceeding we demonstrate that through generating the Gaussian momentum-smeared quark source, excited state contaminations in quasi-PDF matrix elements on the lattice are under control.

\section{PDF in LQCD Calculations}

The definition of the light-cone quark unpolarized PDF, as in parton physics, is

$$
q(x, \varepsilon)=\int \frac{d \xi^{-}}{4 \pi} e^{-i x \xi^{-} P^{+}}\left\langle P\left|\bar{\psi}\left(\xi^{-}\right) \gamma^{+} \exp \left\{-i g \int_{0}^{\xi^{-}} d \eta^{-} A^{+}\left(\eta^{-}\right)\right\} \psi(0)\right| P\right\rangle,
$$

where $A$ is the QCD gauge field vector potential with the gauge coupling constant $g$, and Lorentz vectors are defined in the light-cone coordinate space. Normally the bare PDF is renormalized in the modified minimum-subtraction $(\overline{M S})$ scheme.

Thus, the PDF is a non-perturbative characteristic of the hadron. The time-dependent correlation function, however, cannot be calculated directly in LQCD without further modifications. One solution is the LaMET, where the light-cone hadron frame is rotated slightly toward the space-like frame with a highly boosted hadron, and corrections from this process can be addressed in the perturbative theory by the QCD factorization. The resulted matrix element, as in the definition of the quasi-PDF $\tilde{q}(x)$, is static instead of dynamic and can be directly simulated on the lattice:

$$
\tilde{q}(x)=\int \frac{d z}{4 \pi} e^{-i x z p^{z}}\left\langle P\left|\bar{\psi}(\vec{z}) \gamma_{t} \Gamma_{\hat{z}}(z, 0) \psi(0)\right| P\right\rangle
$$

where $\Gamma_{\hat{z}}(z, 0)$ is the Wilson link of length $z$, with $\hat{z}$ defined conveniently as the hadron spatial momentum direction. Calculations of PDFs are then reduced to solving quasi-PDF matrix elements on the lattice with a large-momentum hadron.

\section{Large-Momentum Hadrons on the Lattice}

Compared to a hadron at rest, the propagator of a nonzero-momentum hadron on the lattice has a lower signal-to-noise ratio with increased time, as well as reduced excited-state energy gaps. Thus, with the spatial momentum of a nucleon at one to a few $\mathrm{GeV}$ (the same energy scale for a 
reliable extrapolation in the large-momentum limit), one needs a clean ground-state signal extrapolation at small-to-moderate $t$ 's to reduce the effect of a low signal-to-noise ratio on the lattice, since the quality of the nucleon signal worsens relatively fast, shrinking the time window to observe the ground state.

On the other hand, the Gaussian-smeared (GS) gauge-invariant quark source [4] has been widely applied in lattice calculations on various bilinear-quark matrix elements. With a proper tuning, it provides an enhanced overlap with the ground state, thus reduces excited state contaminations by a constant factor throughout the entire time region. However, its amplitude spectrum in the momentum space has a peak at $|\vec{p}|=0$. To shift the peak to the target quark momentum $\vec{p}^{q}$, it's straightforward to add in addition a constant phase vector $\vec{k}$, generating the Gaussian momentumsmeared (GMS) gauge-invariant quark source [5]. In practice, we use the iterative method to apply the GMS quark source: $\psi_{i+1}(x)=\psi_{i}(x)+\alpha \sum_{j} U_{j}(x) e^{i k_{j} \hat{e}_{j}} \psi_{i}\left(x+\hat{e}_{j}\right)$, where $i \leq n_{\text {iter }}\left(n_{\text {iter }}\right.$ as the number of smearing iterations). The modified quark source has been shown greatly improves the accuracy of the fitted ground-state nucleon energy with a boosted momentum.

\section{Ground-state Extractions of Nucleon Quark Quasi-PDF Matrix Elements}

Lattice calculations of unpolarized and polarized quasi-PDF matrix elements are done using the Wilson clover quark on a dynamical $N_{f}=2+1+1$ HISQ [6] ensemble generated by MILC collaboration, with one step hypercubic (HYP) smearing [7]. The ensemble contains degenerated up/down quark masses, a strange and a charm quark mass. The lattice spacing is $a \approx 0.09 \mathrm{fm}$, the sea pion mass is $M_{\pi} \approx 310 \mathrm{MeV}$, and 1152 measurements of each matrix element are done on 288 configurations, with four nucleon sources imposed at different time locations on each configuration. We set the valence up/down quark mass degenerate to the strange quark mass, yielding a valence pion mass $m_{\pi} \approx 670 \mathrm{MeV}$, to enable and compare fits including as few as one state in the fitting function.

Matrix elements for unpolarized $(\tilde{q})$ and polarized $(\Delta \tilde{q})$ isovector nucleon valence PDFs are

$$
\begin{aligned}
\tilde{u}-\tilde{d} & =\left\langle P\left|\bar{u}(z) \gamma_{t} \Gamma_{\hat{z}}(z, 0) u(0)-\bar{d}(z) \gamma_{t} \Gamma_{\hat{z}}(z, 0) d(0)\right| P\right\rangle, \\
\Delta \tilde{u}-\Delta \tilde{d} & =\left\langle P\left|\bar{u}(z) \gamma_{z} \gamma_{5} \Gamma_{\hat{z}}(z, 0) u(0)-\bar{d}(z) \gamma_{z} \gamma_{5} \Gamma_{\hat{z}}(z, 0) d(0)\right| P\right\rangle,
\end{aligned}
$$

where the nucleon is boosted along the $z$ direction. For polarized PDFs the nucleon states are also spin-polarized.

The GMS quark sources have been applied, with the set of Gaussian smearing parameters $\alpha=$ $5, n_{\text {iter }}=100$. The final nucleon states are momentum-projected to $P_{z} \approx 2.2 \mathrm{GeV}$. To estimate overall excited state contributions in the three-point correlator, we look at initial and final nucleon operators with a range of temporal separations $t_{\text {sep }} \in\{6,8,10,12\}$ on the lattice, as well as varying the number of channels in the fitting function, including up to the second-excited state. To conclude ground states are extracted out as reliable as one would expect, we compare the fitted ground-state energies among all fits and show that, in our case, the fitting module saturates the data sample, 
meaning any added free parameter would not gain any additional information from the data set.

The fits are done using the Bayesian technique, with loose priors to avoid any over-constraining on the fits. We perform simultaneous $N$-state fits of two- and three-point correlators: for $N=2,3$, data at various $t_{\text {sep }}$ 's are included up to the smallest $t_{\text {sep }}=6$; for $N=1$, data at one of $t_{\text {sep }}=8,10,12$ are included. Nucleon states are projected positive-parity eigenstates. Fitting functions on two- and three-point correlators are

$$
\begin{aligned}
& C_{2 p t}(t)=\sum_{n=1}^{N} A_{n}^{2} e^{-E_{n} t}, \\
& C_{3 p t}(t)=\sum_{n, m=1}^{N} V_{n m} A_{n} A_{m} e^{-\left(E_{n}-E_{m}\right) t-E_{m} t_{s e p}},
\end{aligned}
$$

where $N$ notes the highest excited-state energy level in an $N$-state fit, and $A_{n}^{2}=\left|\left\langle E_{n} \mid P\right\rangle\right|^{2} /\left(2 E_{n}\right)$. Besides, we enable the option to either include or exclude the term of $V_{N N}$ in our fits, independent of other terms.
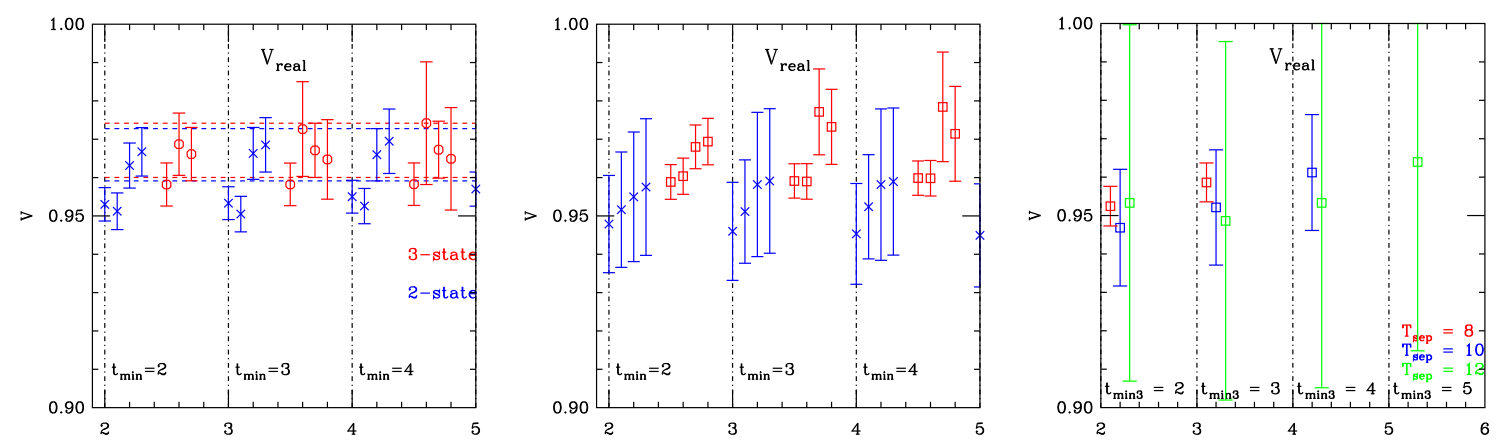

Figure 1: Fitted real unpolarized ground-state nuclear nucleon matrix elements $V_{11}$ at $z=0$. The left plot compares between 3-state fits excluding the $V_{33}$ term (points in red) and 2-state fits including the $V_{22}$ term (points in blue), where the fits include three-point correlators at $t_{\text {sep }}=\{6,8,10,12\}$. The middle plot compares between 2-state fits excluding the $V_{22}$ term (points in red) and including the $V_{22}$ term (points in blue), where the fits include three-point correlators at $t_{\text {sep }}=\{8,10,12\}$. Three panels in the left and middle plots separate fitting ranges of the nucleon two-point correlator at the source end to be $t_{\min }=2,3,4$ from left to right, and each panel shows three-point correlator fitting ranges $\left[t_{\min 3}, t_{\text {sep }}-\right.$ $\left.t_{\min 3}\right]$ at $t_{\min 3}=0,1,2,3$ from left to right for each set of fits. The right plot shows the 1-state fits of $V_{11}$, with three-point correlators at $t_{\text {sep }}=8$ (points in red), 10 (points in blue), and 12 (points in green). Four panels in this plot separate fits from three-point correlator fitting ranges $\left[t_{\min 3}, t_{s e p}-t_{\min 3}\right]$, with $t_{\min 3}=2,3,4,5$ from left to right, all at the same and fixed $t_{\min }=7$.

Figure 1 compares the fitted real unpolarized ground-state nuclear nucleon matrix elements $V_{11}$ at $z=0$ among fits with various $N$ 's and $t_{\text {sep }}$ combinations. Due to the symmetry, $V_{11}$ is real in this case. The left plot shows both $N=2$ and $N=3$ fits at all four $t_{\text {sep }}$ 's, in which the $V_{22}$ term is included while the $V_{33}$ term is excluded throughout. Dotted lines show the picked fit of $V_{11}$ with the error bar, where the fitting window at the source end in the 2-state fit is $t_{\min }=4$ (for the two-point correlator), $t_{\min 3}=2$ (for the three-point correlator), and that in the 3 -state fit is $t_{\min }=3, t_{\min 3}=2$. Confidence levels in both sets of fits are good $(\geq 0.5)$ when $t_{\min }, t_{\min 3} \geq 1$. From 
this plot, one can tell that 2-state and 3-state fits are consistent with each other within errors. We also exaggerated our fits to further include the $V_{33}$ term, but didn't see any benefit from this, given that only error sizes were larger. This indicates that including up to $N=3$ is already saturating the excited-state contributions from current data set. The middle plot shows the fitted $V_{11}$ from two sets of 2-state fits both using the three-point correlator data at three $t_{\text {sep }}$ 's (excluding data at $t_{s e p}=6$ ), and the fitting function either including or excluding the $V_{22}$ term. The right plot shows the result from 1-state fits, again at various three-point correlator fitting ranges of $\left[t_{\min 3}, t_{\operatorname{sep}}-t_{\min 3}\right]$ with $t_{\min 3} \in[2,5]$ and separate $t_{\text {sep }}=8,10,12$ (shown in red, blue, and green). The 1-state fits of this and all other operators share the same two-point correlator fitting window at $t_{\min }=7$, where excited-state contributions become negligible. One can see that fitted $V_{11}$ in 3-, 2- and 1-state fits agree overall within errors, while the 1-state fits have significantly increased error sizes with an increased $t_{\text {sep }}$.
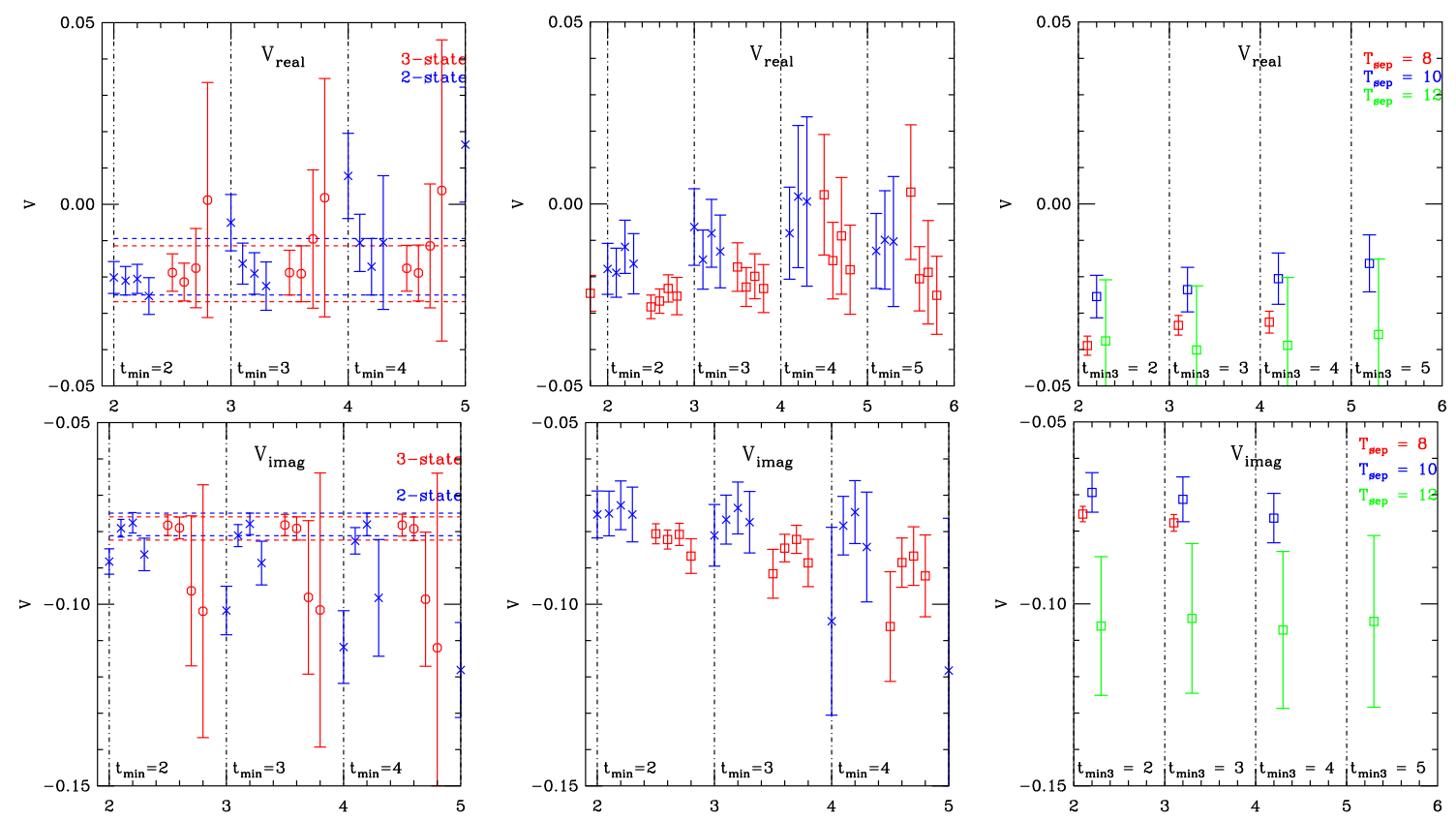

Figure 2: Fitted real (upper row) and imaginary (bottom row) part of unpolarized ground-state nuclear nucleon matrix elements $V_{11}$ at $z=8$. The first row contains the real part of $V_{11}$, and the second row contains the imaginary part. Similar to Figure 1, the left column compares between 3-state fits excluding the $V_{33}$ term (data in red) and 2-state fits including the $V_{22}$ term (data in blue) from data at all four $t_{\text {sep }}$ 's; the middle column compares between 2-state fits excluding the $V_{22}$ term (data in red) and including the $V_{22}$ term (data in blue) from data at $t_{s e p}=\{8,10,12\}$; the right column shows the 1-state fits at $t_{\text {sep }}=8$ (points in red), 10 (points in blue), and 12 (points in green). Again, three(four) panels in the left and middle plot separate the fitting range of nucleon propagators at the source end to be $t_{\min }=2,3,4(, 5)$ from left to right, and each panel shows the three-point correlator fitting range $\left[t_{\min 3}, t_{\operatorname{sep}}-t_{\min 3}\right]$ at $t_{\min 3}=0,1,2,3$ from left to right for each set of fits. Four panels in the right plot separate $t_{\min 3}=2,3,4,5$ from left to right.

Fits on unpolarized quasi-PDF non-local operators are done using the same strategy. Figure 2 shows the fitted $V_{11}$ of the operator with a Wilson-link length $8(z=8)$. Similar to the local operator at $z=0$, picked 2-state and 3-state fits in each plot in the left column are consistent within errors, and they both agree with the 1-state fit at $t_{\text {sep }}=12$ within $1.5 \sigma$. On the other hand, 3-state fits of the imaginary part of $V_{11}$ have notably increased errors to over $20 \%$ at $t_{\min 3} \geq 2$, since the statistical 
errors in the three-point correlator grow more significant with an increased $t_{s e p}$, compared with those of the local operator. Thus the picked 3-state fit here has $t_{\min 3}=1$.
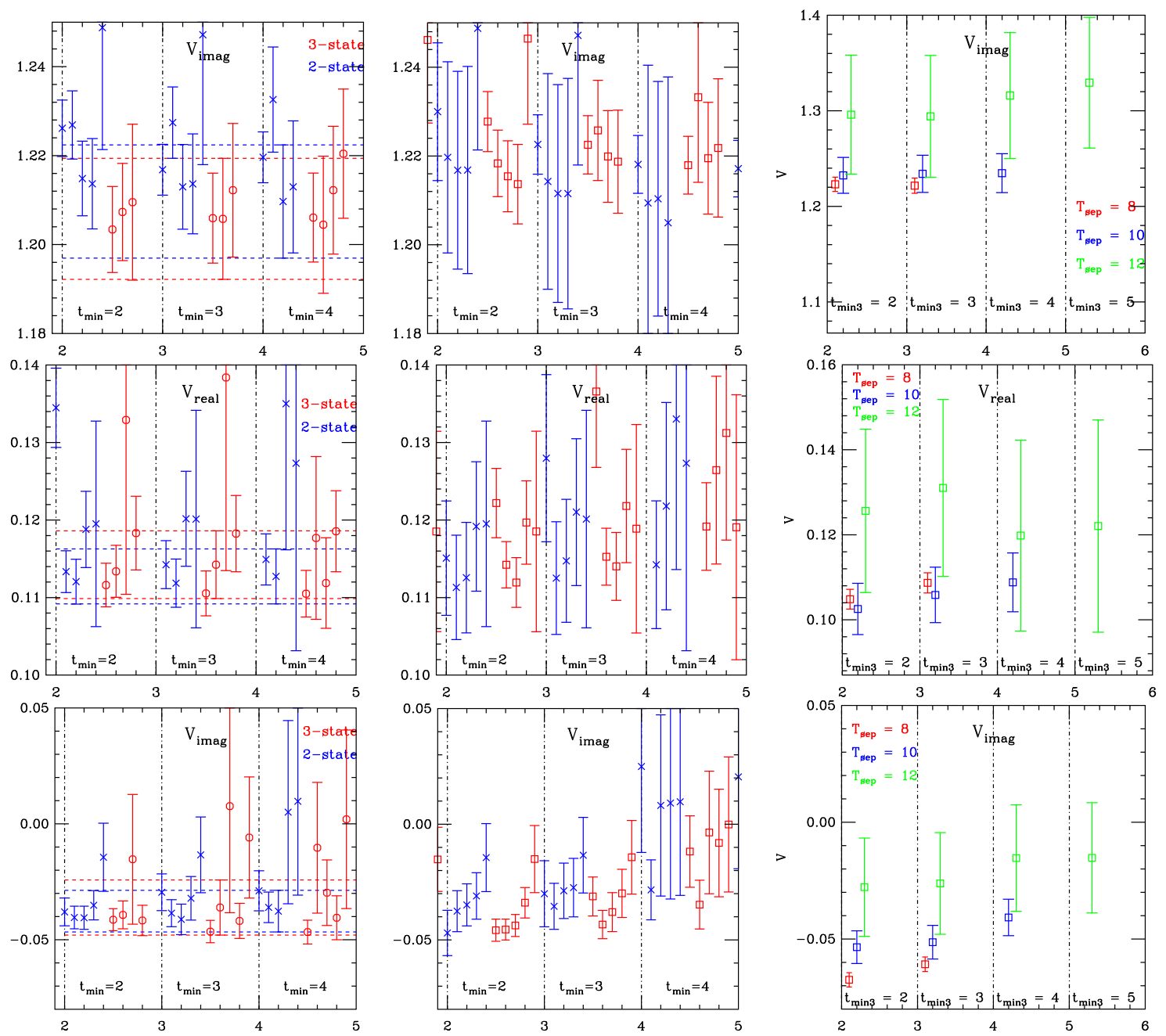

Figure 3: Fitted polarized ground-state nuclear nucleon matrix elements $V_{11}$ at $z=0$ and 8 . The first row contains the imaginary part of $V_{11}$ of the local operator at $z=0$, and the second and third row contains the real and imaginary part of $V_{11}$ of the non-local operator at $z=8$. Plots in three columns are arranged in the same patten as those for the unpolarized quasi-PDF operators.

Same set of fits are also done on the polarized quasi-PDF matrix elements at $z=0,8$, as shown in Figure 3 for the fitted $V_{11}$. The matrix element calculated in this case has the spin-projection operator $\gamma_{x} \gamma_{y} \gamma_{t}=-i \gamma_{z} \gamma_{5}$. Again 2- and 3-state fits here are consistent within errors, and they agree with the 1-state fit at the largest $t_{\text {sep }}$ within $1.5 \sigma$.

\section{Summary}

We show that with improved quark sources through Gaussian momentum-smearing, excitedstate contaminations in quasi-PDF matrix elements on the lattice, even at a small to moderate 
Euclidean time, can be under control and disentangled as demonstrated in our comprehensive fitting models. The capability of a clear ground state extrapolation is crucial for high-precision lattice calculations of the quasi-PDF, and the quark source with an optimized overlap with the ground state is especially beneficial, since it effectively extends the fitting window at the hadron correlator source end.

\section{Acknowledgement}

We thank the MILC collaboration for sharing their $N_{f}=2+1+1$ HISQ ensemble for this study. The LQCD calculations were performed using the Chroma software suite [8]. This research used facilities of the USQCD Collaboration, which are funded by the Office of Science of the U.S. Department of Energy, and supported in part by Michigan State University through computational resources provided by the Institute for Cyber-Enabled Research. HL, RL, and YY are supported by the US National Science Foundation under grant PHY 1653405 "CAREER: Constraining Parton Distribution Functions for New-Physics Searches".

\section{References}

[1] Feynman, R. P., High Energy Collisions: Third International Conference at Stony Brook, N.Y. Gordon Breach. pp. 237-249. ISBN 978-0-677-13950-0, 1969.

[2] X. Ji, Phys. Rev. Lett. 110, 262002, 2013.

[3] M. Deka et al., Phys. Rev. D91, 014505, 2015.

[4] S. Güsken, Nucl. Phys. B Proc. Suppl. 17, 361, 1990.

[5] Gunnar S. Bali et. al., Phys. Rev. D93, 094515, 2016.

[6] E. Follana et. al. (HPQCD, UKQCD), Phys. Rev. D75, 054502, 2007.

[7] A. Hasenfratz and F. Knechtli, Phys. Rev. D64, 034504, 2001.

[8] R. G. Edwards and B. Joo (SciDAC, LHPC, UKQCD), Nucl. Phys. Proc. Suppl. 140, 832, 2005 